\title{
Introduction to "Experimental Approaches to the Study of Child Language: A Cross-Linguistic Perspective"
}

\author{
Peng Zhou ${ }^{1} \cdot$ Stephen Crain ${ }^{2} \cdot$ Michael C. W. Yip ${ }^{3}$ \\ Published online: 19 September 2018 \\ (c) Springer Science+Business Media, LLC, part of Springer Nature 2018
}

There is an increasing interest in using experimental approaches to investigate children's acquisition of semantic and pragmatic knowledge. These areas are now being explored both in Indo-European Languages and also in Asian languages. Such cross-linguistic studies are essential to our understanding of the universal properties of language, as well as the ways in which languages can differ. The goal of this special issue is to highlight this cross-linguistic and experimental perspective on children's use of semantic and pragmatic knowledge in sentence production and comprehension.

This issue contains nine papers that can be divided into four major themes: children's scope assignment, the acquisition of language specific phenomena, the relation between children's linguistic knowledge and their non-linguistic abilities, and the use of $w h$-words by typically developing (TD) children and by children with language delay.

\section{Children's Scope Assignment}

The first three papers take a cross-linguistic perspective on the investigation of children's interpretation of sentences containing two logical operators. Koring, Meroni and Moscati looked at Dutch-speaking children's early assignments of scope, Gao, Thornton, Zhou and Crain explored related phenomena in child Mandarin, and Pagliarini, Crain and Guasti explored these phenomena in child Italian. The findings suggest that children initially exhibit similar interpretative patterns regardless of the local language they are being exposed to. The findings support the constraint on language learnability known as the Semantic Subset Principle (Crain et al. 1994). This principle anticipates that children's initial scope assignment corresponds to the interpretation that makes sentences true in the narrowest range of circumstances, even when this is not the interpretation assigned by adults in the same linguistic community. The initial 'subset' assignment of scope guarantees that children will be able to converge on the

Peng Zhou

zhoupeng1892@mail.tsinghua.edu.cn

1 Department of Foreign Languages and Literatures, Child Cognition Lab, Tsinghua University, Beijing 100084, China

2 ARC Centre of Excellence in Cognition and Its Disorders and Department of Linguistics, Macquarie University, Sydney, NSW 2019, Australia

3 Department of Psychology, The Education University of Hong Kong, Hong Kong, China 
scope assignments of adult speakers, without recourse to negative evidence, i.e., based on positive evidence in the input from adults.

\section{The Acquisition of Language Specific Phenomena}

The papers by Hu, Guasti and Gavarró and by Yang, Shi and Xu explored children's acquisition of language specific phenomena. Hu et al. studied Mandarin-speaking children's acquisition of topicalization in the context of the debate of whether or not topic structures in Mandarin are derived via movement (see e.g., Huang et al. 2009; Li and Thompson 1981; Xu and Langendoen 1985). On the basis of the findings, Hu et al. proposed that children initially adopt a non-movement analysis of topic structures. Yang et al. investigated Mandarin-speaking children's understanding of grammatical aspect. Unlike many Indo-European languages, Mandarin does not have tense markers, but it has a rich aspectual system. The results showed that 30-month-old Mandarin-speaking children are already sensitive to the distinction between the durative aspect marker-zhe and the perfective aspect marker-le.

\section{The Relation Between Children's Linguistic Knowledge and Their Non-linguistic Abilities (e.g., Conceptual Knowledge)}

The papers by Shi and Zhou and by Chu and Minai examined the interaction between children's linguistic knowledge and their non-linguistic abilities. Shi and Zhou investigated Mandarin-speaking children's use of possessive constructions to express possessive relations. The findings showed that, by 4 years of age, Mandarin-speaking children correctly understand and produce the typical possessive DE constructions in Mandarin. Children at age 3, however, are still at a transitional stage where they use noun-noun compounds to represent possessive relations. Chu and Minai looked at the interaction between children's comprehension of demonstratives and their non-linguistic cognitive abilities involving Theory of Mind and Executive Function. Both English- and Mandarin-speaking children were tested, and the results showed that children's knowledge of demonstratives is related to the development of non-linguistic cognitive abilities, regardless of the language they are acquiring.

\section{Wh-Words in Both TD Children and Children with Language Delay}

The last two papers investigated the interpretation of $w h$-words by both TD children and children with language delay. Huang, Zhou and Crain focused on TD Mandarin-speaking children's comprehension of wh-words and found that 5-year-old children already exhibit adult-like knowledge of the semantics and pragmatics of wh-words. de Villiers, Ning, Liu, Zhang and Jiang compared the acquisition of paired $w h$-questions by TD children and by children with language delay. They interpreted the findings of their study as evidence that language-delayed Mandarin-speaking children may suffer from a delay in accessing the requisite semantic knowledge when they attempt to answer paired wh-questions. 


\section{Conclusion}

The questions raised in these papers are all important questions for our understanding of children's mastery of language, representing the latest trends and advancements in this field. This research has benefited from the growing interest in combining cross-linguistic and experimental approaches to child language. It is worth noting, in this regard, that many of the studies reported in this volume adopted research methods that have been widely used in previous studies to investigate the acquisition of language by young TD children, including the Truth Value Judgment Task, the Elicited Production Task (see Crain and Thornton 1998), and the Intermodal Preferential Looking Paradigm (see Golinkoff et al. 1987). As the studies reported here suggest, these tasks are also valuable tools in assessing children with language disorders. We would also highlight the fact that the majority of the studies included in this volume are theory-motivated and, therefore, they provide good examples of convergence between child language acquisition and linguistic theory. We believe that theory-motivated child language research represents a healthy direction for the field to take, a direction that promises to contribute to our understanding of the nature of human language. We are confident that this special issue will make a valuable contribution to the field and will inspire more exciting future research.

\section{Compliance with Ethical Standards}

Conflict of interest The authors declare that they have no conflict of interest.

\section{References}

Crain, S., Ni, W., \& Conway, L. (1994). Learning, parsing and modularity. In C. Clifton, L. Frazer, \& K. Rayner (Eds.), Perspective on sentence processing. Hillside, NJ: Lawrence Erlbaum.

Crain, S., \& Thornton, R. (1998). Investigations in universal grammar: A guide to experiments on the acquisition of syntax and semantics. Cambridge, MA: MIT Press.

Golinkoff, R. M., Hirsh-Pasek, K., Cauley, K. M., \& Gordon, L. (1987). The eyes have it: Lexical and syntactic comprehension in a new paradigm. Journal of Child Language, 14, $23-45$.

Huang, C.-T. J., Li, Y.-H. A., \& Li, Y. (2009). The syntax of Chinese. Cambridge: Cambridge University Press.

Li, C. N., \& Thompson, S. A. (1981). Mandarin Chinese: A functional reference grammar. Berkeley: University of California Press.

Xu, L., \& Langendoen, D. T. (1985). Topic structures in Chinese. Language, 61, 1-27. 